\title{
Evaluación Farmacocinética en dos Formas Orales de Dosaje de Cloramfenicol en Niños con Fiebres Tíficas
}

\author{
Q.F. María E. Bravo R. ${ }^{1}$; Dr. Isidoro Horwitz C. ${ }^{2}$; Q.F. Angélica Sánchez V. ${ }^{3}$; \\ Q.F. Aquiles Arancibin $O^{3}$ \\ Pharmacokinetic Evaluation of Two Forms of Chloramphenicol \\ for Oral use in Children with Typhoid Fever
}

\begin{abstract}
19 patients of both sexes, aged $9.3 \pm 3.0(\bar{X} \pm$ S.D.) years with bacteriologically confirmed typhoid or paratyphoid $B$ fever were enrolled in this study and distributed in two treatment groups: Patients of Group [ $(N=11)$ received Cloramphenicol (CAP) palmitate (suspension) $50 \mathrm{mg} / \mathrm{kg}$ body weight (BW) in two daily doses; Group ${ }^{-11}$ patients $(\mathrm{N}=8)$ received $\mathrm{CAP}$-crystalline (capsules) in the same doses and frecuency of administration. Several capillary blood samples were taken through twelve hours after the administration of the first dose. Frec plasma C.AP was measured radioenzymaticaly. Phatmacokinetic parameters were calculated according to the one compartment model. Significant differences in the plasma levels of the two pharmaceutical preparations at 30 minutes $(\mathrm{P}<0.001)$ were found, being ligher those of crystalline CAP. Useful therapeutic concentrations were obtained with both CAP preparations. No significant differences wete found in the pharmacokinetic parameters. The clinical restults were equally good with both preparations. Both CAP palmitate and crystalline base have the same biosvailability and are suitable for the treatment of enteric fevers.

(Key words: Chloramphenicol. Oral Paimitate Suspension. Crystaline. Pharracokinetics. Typhoid Fever).
\end{abstract}

Las infecciones entéricas y en especial las producidas por Salmonella del grupo tífico $y$ paratífico se manifiestan en Chile con una morbilidad elevada $y$ con tendencia a un aumento continuo. La tasa de morbilidad en el último año llegó a $120 \times 100.000$ habitantes en todo el país y a 180.000 o más en la Región Metropolitana ${ }^{1}$.

Diversos antibióticos (cloramfenjicol, ampicilina) en distintos regímenes terapéuticos y diferentes agentes quimioterapéuticos (furazolidona, sulfametoxazol-trimetoprim) han sido usado en el tratamiento de las fiebres entéricas por nuestro grupo. Los resultados clínicos con todos ellos, han sido más o menos comparables con la excepción de los pacientes tratados con ampicilina, que en promedio demoran un mayor número de días en tomarse afebriles. El cloramfenicol (CAF) ha con-

1. Instituto de Nutrición y Tecnología de los Alimentos (INTA) Universidad de Chile.

2. Departamento de Pediatría, Facultad de Medicina. Universidad de Chile.

3. Departamento de Ciencias Farmacológicas, Facultad de Ciencias Farmacéuticas.

Este trabajo fue financiado por el Proyecto $\mathrm{N}^{\circ}$ 678-8355 del Depto. de Desarrollo de la Investigación y Bibliotecas. Universidad de Chile. timuado siendo la droga de alección porque es fácil de administrar, de bajo costo y porque sus efectos tóxicos en los órganos eritropoiéticos no han sido registrados en nuestra población.

El cloramfenicol, para la administración oral, está disponible en cápsulas conteniendo 2500 $500 \mathrm{mg}$ de la base cristalina y como palmitato en una suspensión de $125 \mathrm{mg}$ por $5 \mathrm{ml}$. En los niños, la administración de las cápsulas puede ser difícil debido al tamaño y al sabor amargo. En cuanto al ester palmitado, se ha excluido del tratamiento de fiebres entéricas basado en la impresión clínica de que esta forma farmacéutica es de baja efectividad.

Publicaciones farmacocinéticos recientes de CAF efectuados en pacientes con meningitis bacteriana han demostrado que el palmitato tiene una excelente biodisponibilidad cuando se compara con el succinato ${ }^{2}$. Esto nos condujo a iniciar estudios tendientes a reevaluar la utilidad del palmitato de cloranfenicol en el tratamiento de las fiebres entéricas.

Este trabajo se realizó con los propósitos de comparar, mediante parámetros farmacocinéticos, la biodisponibilidad de dos formas farmacéuticas (cápsulas conteniendo $250 \mathrm{mg}$ de CAF cristalino versus palmitato de CAF en sus. pensión conteniendo $125 \mathrm{mg}$ por $5 \mathrm{ml}$ ) y estudiar 
la farmacocinética del CAF en pacientes con fiebres entéricas.

\section{PACIENTES Y METODOS}

E] estudio fue realizado en 29 pacientes de ambos sexos, hospitalizados en la Unidad de Infecciosos del Hospital Roberto del Río en Santiago, con el diagnóstico de fiebres tificas o paratificas, entre Noviembre de 1982 y Junio de 1983. En todos ellos el diagnóstico fue bacteriológicamente certificado por cultivo de sangre, médula ósea o ambos. Los pacientes fueron tratados con cloramfenicol de acuerdo con el régimen usado en dicho hospital: $50 \mathrm{mg} \mathrm{x} \mathrm{kg} \mathrm{x}$ día, fraccionado cada 12 hrs., durante el período febril, dosis que es reducida a $25 \mathrm{mg} \times \mathrm{kg}$ ' $\mathrm{x}$ día cuando el paciente ha permanecido afebril por 48 horas ${ }^{3}$, situación que ocurre generalmente al $6^{\circ}$ dia. Los pacientes fueron tratados durante el número total de 14 días.

Los pacientes fueron alternativamente asignados de acuerdo a su fecha de admisión al grupo que recibió cápsulas o al tratado con suspensión de palmitato, con una distribución final de 11 niños que recibieron la suspensión de palmitato y 8 que recibieron cápsulas. El número inicial de 29 sujetos se redujo a 19 porque 5 pacientes fueron excluidos por haberse obterido de ellos un insuficiente numero de muestras sanguineas $y$ otros 5 pacientes cuyas concentraciones plasmáticas no deeayeron hasta las 12 horas, fueron agrupados por separado.

Los pacientes de ambos grupos recibieron paracetamol como antipirético en dosis de $10 \mathrm{mg} / \mathrm{kg}$ cada vez que tuvieron fiebre alta. No hubo registro de los medicamentos ingeridos antes del ingreso al hospital. Antes de la primera dosis de CAF, se extrajo una muestra de sangre para las pruebas de rutina (protejnas del plasma, bilirrubina, creatinina, nitrógeno ureico y transaminasas pirúvica y oxalacética) y para la determinación del nivel basal o tiempo cero de CAF (en un paciente tratado con palmitato, las pruebas de laboratorio no fuecon efectuadas). Después de la administración de la primera dosis de CAF, se tomaron muestras de Sangre $(50 \mu \mathrm{l})$ en los siguientes $30,60,120,180,240,360$ y 720 minutos. Las muestras fueron obtenidas por punción capilar, recogidas en tubos heparinizados y guardadas a $-70^{\circ} \mathrm{C}$ hasta el momento del análisis.

E1 tratamiento comenzó entre 5 y 18 días $(11,7 \pm 4,0)$ después del comienzo de la enfermedad en los pacientes tratados con palmitato $y$ entre 6 y 16 dias $(9,3 \pm 3,4)$ en aquellos tratados con cápsulas.

Las muestras de sangre fueron analizadas sin conocimiento, por parte de los analistas, de la forma farmacéutica que estaban recibiendo los pacientes.

El cloramfenicol libre fue medido por el método de radioenzimático de Lietman et al. ${ }^{4}$. Este método en nuestra experiencia resultó tener una sensibilidad de hasta $0,5 \mu \mathrm{g} / \mathrm{ml}$ de $\mathrm{CAF}$ en plasma. La reproducibilidad medida en alícuotas de plasma conteniendo $20 \mathrm{\mu g} / \mathrm{ml}$ mostró un coeficiente de variación de $4,4 \%$ y la comparación con el método cromatográfico de Nílsson-Ehle ${ }^{5}$, mostró un coeficiente de correlación de 0,98 . Las determinaciones de $\mathrm{CAF}$ plasmático fueron efectuadas en duplicado y estandares frescos de CAF en plasma fueron incluidos cada vez que se hicieron las determinaciones de los pacientes.

El protocolo de estudio fue aprobado por el Comité de Etica del Instituto de Nutrición y Tecnologia de los Alimentos.

Análisis Farmacocinético. Para cada paciente los datos de concentración plasmática fueron ajusta. dos a la siguiente función:

$$
C=\frac{F X o k a}{V d(k a-K}\left(e^{-k t}-e^{-k a t}\right)
$$

donde: $C=$ Concentración de $\mathrm{CAF} ; F=$ fracción de la dosis realmente absorbidos; $X o=$ dosis administrada; $V d=$ volumen de distribución; $K=$ constainte de velocidad de eliminación de primer orden; $k e=$ constante de velocidad de absorción de primer orden; $t=$ tiempo despues de la administración.

La constante de velocidad de eliminación $\mathbf{K}$ se determinó por regresión ineal por el método de los cuadrados minimos, usando cuatro puntos de la parte terminal del gráfico del logaritmo natural de la concentración versus el tiempo. La vida media de eliminación ( $t / 2)$ fue calculado usando la ecuación:

$$
t I / 2=\frac{\ln 2}{K}
$$

Para calcular la constante de velocidad de absorción ka se empleó el método de los residuos.

El tiempo en el cual se alcanzó la concen. tración máxjma denominada $t$ máx se calculó a 
partir de la ecuación:

$$
t \max =\frac{\ln (i k a / k)}{(k a-K)}
$$

El área bajo la curva $(A B C)$ de concentraciones plasmáticas versus tiempo fue calculada por el método de los trapecios.

El volumen de distribución aparente expresado como "Vd area", se determinô por la ecuación:

$$
V d=\frac{d o s i s}{A B C K}
$$

La depuración $(D)$ se calculó a partir de la siguiente ecuación:

$$
D=\frac{\text { dosis }}{A B C}
$$

Para ambos, Vd area y depuración, se partió del supuesto que $\mathbf{F}$ es igual a 1 .

Para valorar las diferencias entre los grupos, se usó la prueba $t$ de Student, considerándose significativos los valores de $\mathrm{p}<0,001$.

\section{RESULTADOS}

La curva de concentración plasmática de CAF en el tiempo se expresa en la Figura 1, donde se puede ver que a los 30 y 60 minutos las concentraciones promedios de CAF fueron de 2,0 y 5,2 Hg $\times \mathrm{ml}$ respectivamente para los pacientes que recibieron palmitato de cloramfenicol, mientras que para aquellos que recibieron las cápsulas, estos valores fueron de 9,6 y $9,4 \mu \mathrm{g} \mathrm{x} \mathrm{ml}$. Estas diferencias. son estadísticamente significativas ( $p<0,001$ a los 30 minutos y entre 0,05 y 0,10 a los $60 \mathrm{~min}$.). A partir de los $180 \mathrm{~min}$. , las concentraciones plasmaticas declinaron paralela. mente siendo más alta con palmitato de cloramfenicol, sin embargo, estas diferencias no fueron significativas. La concentración máxima, "Cmax", se produjo entre 2 y 3 horas después de la ingestión. En los pacientes que recibieron palmitato de cloramfenicol se observó mayores concentraciones plasmáticas entre la 2a. y 3a. hora que en los pacientes tratados con las cápsulas.

Los parámetros farmacocinéticos (promedio \pm desviación estándar) se presentan en la Tabla 1. Se puede ver que no hay diferencias significa. tivas.

Todos los pacientes tuvieron buena evolución clínica. En el grupo tratado con paimitato de cloramfenicol la fiebre desapareció en $3,7 \pm 2$ dias de tratamiento, en los ninos que recibieron cápsulas esto ocurrió a los $4,1 \pm 1,9$ días, diferencia que no es significativa.

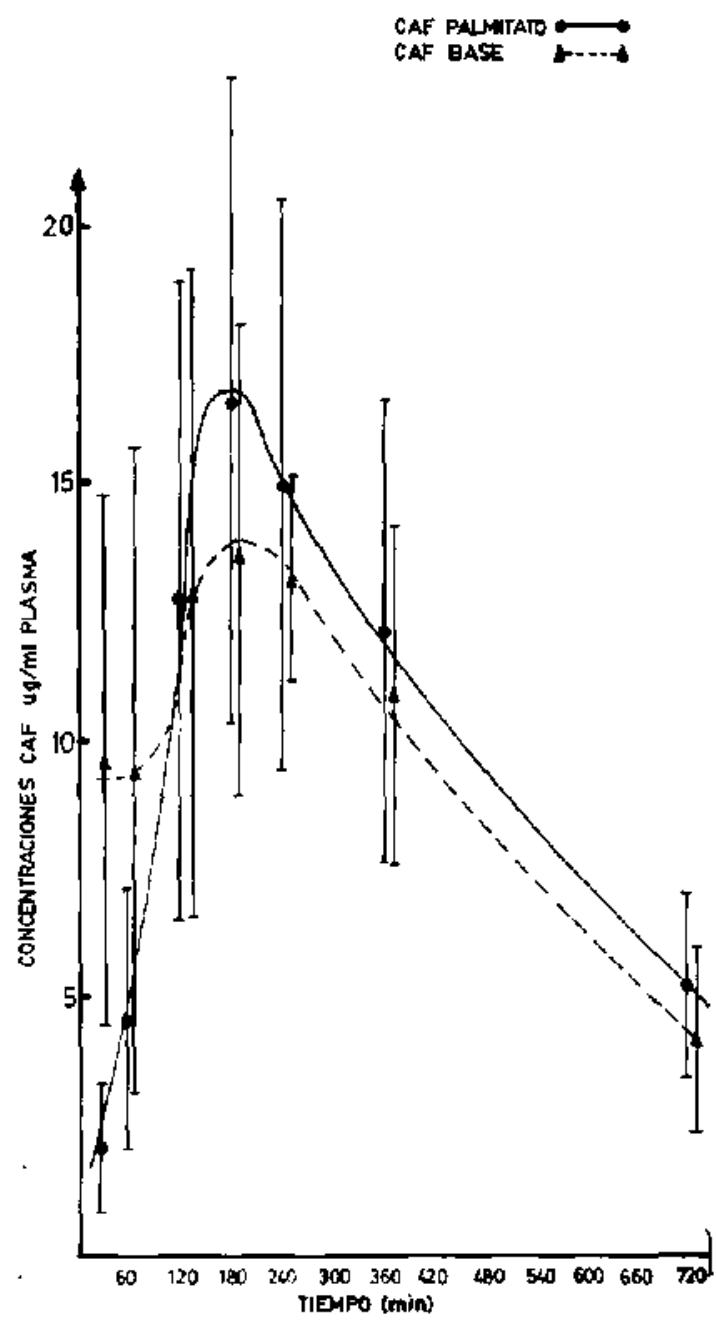

Figura 1: Concentraciones plasmáticas (promedio $\pm \mathrm{DE}$ ) de cloramfenicol después de la administración oral de $50 \mathrm{mg} \times \mathrm{kg}$ de peso corporal cada 12 horas Para evitar la superposición de las curvas de los valores obtenidos con las cápsulas se desplazaron hacia la izquierda en la Figura.

\section{DISCUSION}

En la revisión de la litęratura hay una sola comunicación en la cual se hayan medido con. centraciones plasmáticas en niffos con fiebres tificas ${ }^{6}$. Recientemente en los paises desarrollados se ha extendido el uso del cloramfenicol pero la información farmacocinética está especialmente relacionada a meningitis por hemóphilus influenzae resistente a ampicilina y a otras enfermedades infecciosas. Las fiebres tíficas son sólo un problema de los países subdesarrollados y el cloramfenicol es el antibiótico de elección para estas enfermedades.

Las diferencias en las concentraciones plasmá. ticas alcanzadas a los 30 y 60 minutos pueden ser 
Tabla 1.

Parámetros farmacocinéticos de cloramfenicol libre en niños con fiebres tíficas*

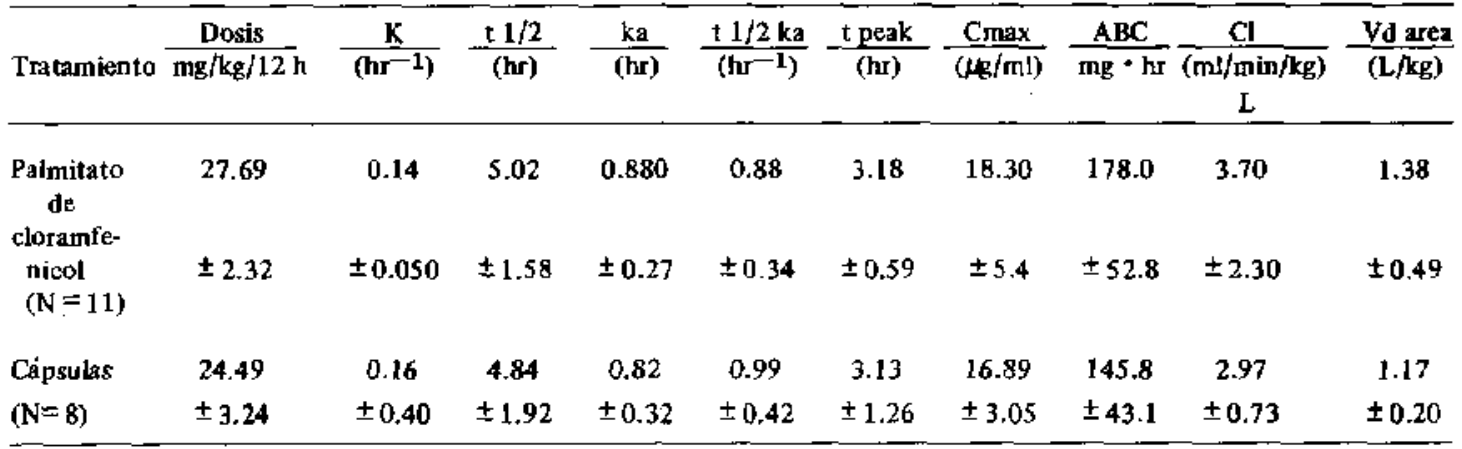

*Todos los valores son promedio \pm desviación estándard.

explicadas sobre la base de que el palmitato de cloramfenicol debe ser hidrolizado antes de ser absorbido.

En general el margen de variación de las concentraciones plasmáticas de cloramfenicol fue mayor con la suspensión de palmitato. La detec. ción de niveles menores de CAF en el plasma en la primera hora, y mayores en las 2 y 3 horas siguientes, con palmitato que con capsulas, aunque no significativa, es muy diferente a lo descrito por Glasko y cols. en sujetos adultos, en quienes ha comunicado valores iniciales más altos con palmitato de cloramfénicol cristalino, aunque la velocidad de decaimiento después de 4 horas fue la misma para las dos preparaciones?

En este estudio las concentraciones plasmá. ticas fueron similares a las descritas por Tou. manen et al. ${ }^{8}$ y Pickering et al..$^{9}$ en lactantes $y$ nijos con otras enfermedades infecciosas. Se encontró una gran variabilidad en la vida media y debido que este parámetro es usado para establecer la frecuencia de administración nos parece interesante enfatizar que ésto ocurre con ambas formas de dosificación, siendo mayor con palmitato. La concentraciŏn máxima (Cmáx) fue alcanzada entre 2 y 4 horas con el palmitato y entre 2 y .5 horas con las cápsulas, to que concuerda con lo informado en la literatura. En sólo dos pacientes, las concentraciones plasmá. ticas alcanzaron valores que se han descrito como tóxicos $(26 \mu \mathrm{g} / \mathrm{ml})$ en el "t mảx.".

En cuanto al volumen aparente de distri. bución Vd area $(\mathrm{L} / \mathrm{Kg})$ está de acuerdo con lo ya establecido por Sack $^{10}$ y Friedman ${ }^{11}$. La biodisponibilidad medida usando el área bajo la curva tuvo un rango similar de variación para ambas formas farmacéuticas; dato que prueba que ambas formas farmacéuticas son jgualmente útiles para tratar fiebres entéricas.

Un grupo de cinco pacientes (Tabla 2) se comportó de manera diferente alcanzando la concentración máxima a las 6 horas y declinando tan lentamente que no fue posible calcular los parămetros farmacocinéticos. De éstos, cuatro pertenecían al grupo tratado con palmitato.

Hallazgos similares ha sido descrito por otros autores (Tabla 3) no habiendo hasta el momento una adecuada explicación para este fenómeno.

\section{Tabia 2.}

Concentración máxima y tiempo en el cual ésta se logró en los pacientes que mostraron una concentración fasmacocinética distinta.

\begin{tabular}{|c|c|c|c|}
\hline \multirow[t]{2}{*}{ TRATAMIENTO } & & CInax & t peak \\
\hline & Pacientes & $(\mathrm{Hg} / \mathrm{mD})$ & (hr) \\
\hline \multirow{4}{*}{$\begin{array}{l}\text { Cloramfenicol } \\
\text { cápsulas }\end{array}$} & S.P.O. & 20.5 & 6.42 \\
\hline & E.R.G. & 13.5 & 6.08 \\
\hline & Y.N.N. & 15.0 & 5.92 \\
\hline & J.D.V. & 21.2 & 5.75 \\
\hline \multirow{3}{*}{$\begin{array}{l}\text { Palmitado de } \\
\text { cloramfenicol }\end{array}$} & \multicolumn{2}{|c|}{ Promedio \pm 17.6} & 6.04 \\
\hline & D.S. & 3.9 & 0.29 \\
\hline & D.C.C. & 16.0 & 4.50 \\
\hline
\end{tabular}

\section{RESUMEN}

19 pacientes de ambos sexos de $9,3 \pm 3,0$ (promedio \pm desviación estándar) años, con fie. bre tífica o paratifica bacteriológicamente confirmada fueron incluidos en este estudio $y$ distribuidos en dos grupos de tratamiento. Los niños del Grupo I $(N=11)$ recibieron suspensión de palmitato de cloramfenicol $50 \mathrm{mg} / \mathrm{kg}$ de peso 
Tabla 3.

Resumen de pacientes (pertenecjentes a diferentes grupos clínicos) que muestran un comportamiento farmacocinético diferente

\begin{tabular}{|c|c|c|c|c|}
\hline Referencia & Forma farmacéutica & $\begin{array}{l}\text { Número total } \\
\text { de pacientes } \\
\text { estudiados }\end{array}$ & $\begin{array}{l}\text { No de pacientes } \\
\text { en los cuales } \\
\text { la concentración } \\
\text { plasmática no decayó }\end{array}$ & $\begin{array}{l}\text { \%de los } \\
\text { casos }\end{array}$ \\
\hline Toumanen, 1981 & $\begin{array}{l}\text { Succinato } y \\
\text { palmitato }\end{array}$ & 44 & 10 & 22.7 \\
\hline Sack, 1980 & Succirato & 17 & 1 & 5.9 \\
\hline Kauffman, 1181 & Succinato & 45 & 15 & 33.3 \\
\hline Freedman, 1178 & $\begin{array}{l}\text { Succinato } y \\
\text { palmitato }\end{array}$ & 56 & 8 & 17.0 \\
\hline
\end{tabular}

corporal en dos dosis diarias. Los pacientes del grupo II $(\mathrm{N}=8)$ recibieron cápsulas conteniendo cloramfenicol en la misma dosis y frecuencia. Se tomaron muestras capilares de sangre en distintos tiempos después de la administración de la primera dosis. El cloramfenicol libre se midió por un método radioenzimático: Los parámetros farmacocinéticos fueron calculados de acuerdo con un modelo de un compartimento. Se encontraron diferencias significativas en las concentraciones plasmáticas obtenidas con las dos preparaciones farmacéuticas a los 30 minutos $(\mathrm{p}<0,001)$ siendo mayores con cloramfenicol cápsulas, pero se obtuvieron concentraciones terapéuticas útiles con ambas preparaciones. No ocurrieron diferencias significativas entre los parametros farmacocinéticos. Los resultados clínicos fueron igualmente buenos con ambas formas farmacéuticas.

\section{AGRADECIMIENTOS}

Este trabajo fue realizado con fondos otorgados por el Departamento de Investigación y Bibliotecas de la Universidad de Chile (Proyecto M-678-8355).

Se agradece en forma especial la ayuda técnica de Sergio Andrés Latorre y la excelente labor secretarial de Sra. Viola Lyon.

\section{REFERENCIAS}

1. Ristori, C.: Epidemiología de la fiebre tifoídea en Chile. Boletín de Vigilancin Epidemiológica 8: 8, 1981.
2. Kauffmann, R.E., Thirumoorthi, M.C., Buckley, J.A., Aravind, M.K. Dojani, A.S.: Relative bioavai lability of intravenous chloramphenicol succinate and oral chloramphenicol palmitate in infants and children, J. Pediatr. 99: 963, 1982.

3. Aguiló, C. et al.: Normas de atención pediátricas. 2nd ed. Santiago, Edit. Andrés Bello, 1984.

4. Lierman, P.S. Thomes, T.J., Shaw. W. V.: Chloramphenicol: an enzynological microassay. Antimicrob. Agents. Chemother. 10: 347, 1976.

5. Nilsson-Ehle, I. et al: Determination of chloramphenicol in serum and cerebrospinal flyid with high-pressure liquied chromatography. J. Antim. Chem. 4: 169, 1978.

6. Schuster, A., Guzmán E.: Niveles sanguíneos del cloramfenicol suministrado por diferentes vías. Rev. Chil, Pedjatr. 25: 369, 1954.

7. Gkazka, A.J., Dill, A.W., Kazenko, A., Wolf, M.L. and Cames, E.H.: Physical factors affecting the rate of absorption of chloramphenicol esters. Antibiotics and Chemotherapy 8: 516, 1958.

8. Toumanen, E.I., Powell, K.R., Marks, M.I., Lafereira, C.I, Allemiller, D.H., Sack, C., Smith, A.L.i Oral chtoramphenicol in the treatment of haernophilus influenzae maningitis. J. Pediatr, 99: 968, 1981.

9. Pickering, L.K., Hoecher, J.L., Kramer, W.G., Kohl, S., Cleary, T.G.: Clinicial pharmacology of two chloramphenicl preparations in children: sodium succinate (IV) and palmitate (Oral) esters. J. Pediatr. 96: 757, 1980.

10. Sack, C.M., Koup, J.R., Smith, A.L.: Chbramphenicol pharmacokinetics in infants and young children. Pediatrics 66: 579,1980 .

11. Friedmen, C.A., Levejoy, F.C., Smith, A.L.: Chloramphenicol disposition in infants and children. $J$. Pediatric 95: 1071, 1979. 